\title{
Characterising and Evidencing the Effects of Porosity in Nano-Electrochemistry
}

\author{
Christopher Batchelor-McAuley and Richard G. Compton \\ Physical and Theoretical Chemistry Laboratory, Department of Chemistry, University of \\ Oxford, South Parks Road, Oxford OX1 3QZ, United Kingdom
}

\begin{abstract}
Porosity in nanoparticles and their ensembles can profoundly alter a system's 10 electrochemical behaviour by both changing the number and identity of the available catalytic sites, and through influencing the mass-transport in the vicinity of the electrochemical interface. This review focuses first on recent advancements in characterising porosity and heterogeneity at the nanoscale, and second on developments in electrochemical simulation which provide insight into how a porous architecture can lead to apparent 'catalytic' effects by virtue of altering the mass-transport in the vicinity of the electrode.
\end{abstract}

Keywords: Heterogeneity, Porosity, Electron Microscopy, Mass-transport, Catalysis

\section{Introduction}

Early in the 20th century Langmuir recognised that for heterogeneous catalysis the study of 'real' catalysts was, from a theoretical stand-point, often prohibitive:1

"Most finely divided catalysts, such as platinum black, or activated charcoal, etc., must have structures of great complexity, and it is probable that the atoms are attached to each other in the form of branching chains so that there are hardly any groups of as little as three or four atoms which are as closely packed as they would be in the crystalline solid. In order to simplify our theoretical consideration of reactions on surfaces, let us confine our attention mainly to reactions on plane 
surfaces. If the principles in this case are well understood, it should then be possible to extend the theory to the case of porous bodies."

This has become the 'surface science' ${ }^{2}$ approach which has, since the advent of technologies and methods for creating reproducible single crystal electrodes, ${ }^{3-4}$ driven much of our theoretical understanding of interfacial reactions. From an academic standpoint and in combination with DFT this has been a fertile area of study. At the centre of this surface science approach is the assumption that if the fundamental principles are understood regarding the reactivity of a well-defined plane then it should be possible to extend this knowledge to 'real' (aka porous) materials; this can be achieved by essentially modelling real catalysts as the sum of their parts. However, the fact remains 'real-world' conditions are non-ideal and often utilise relatively poorly defined catalysts. This surface science approach does not account for the possibility that new chemical or catalytic behaviour may arise in structures as they deviate away from ideality. Furthermore - and probably more problematically - it does not answer the practical question of how, once a new or novel nanomaterial has been produced or synthesised, can we determine its chemical or catalytic activity.

As computing power expands, we are no-longer limited to considering simple electrode geometries, or analysing data using overly restrictive analytical approximations; we can now readily numerically model systems of greater complexity; including, diffusion across the surface of cubes ${ }^{5}$, through layers of beads ${ }^{6}$ or inside particles. ${ }^{7}$ Answering how the electrode architecture and structure, at multiple length scales, may effect the electrochemical response has become a more approachable task. This review will focus on two areas of development in the nano-electrochemical field. First, we look at advancements made in characterising non-ideal nanostructures focusing on heterogeneity in nanoparticle populations. Second, we move to consider new results which demonstrate how large ensembles of nanoparticles, as may be employed in a 'drop-cast' experiment, can alter a system's electrochemical behaviour leading to apparent changes in the voltammetric response not necessarily reflecting the sum of the responses of individual isolated particles. 

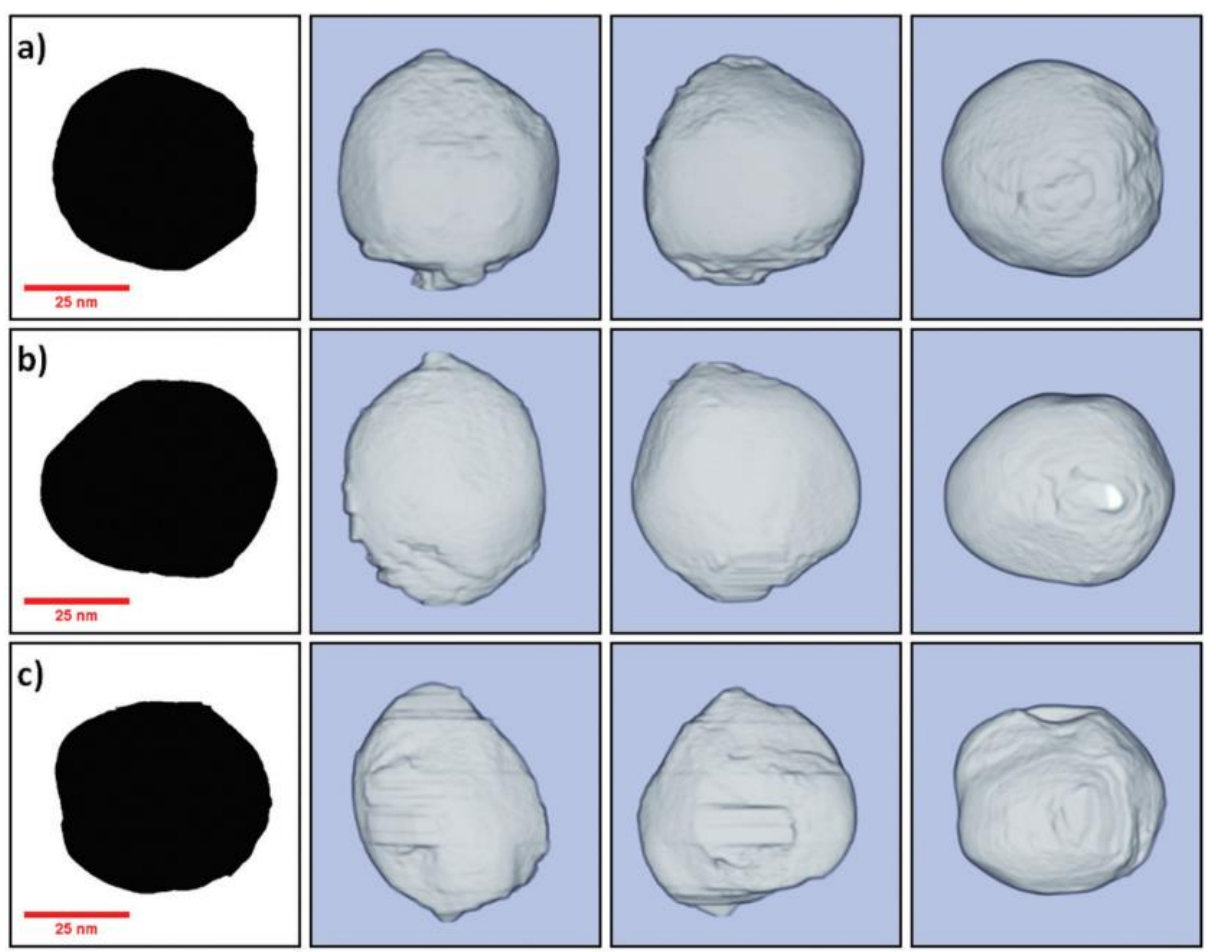

Figure 1: Three ca. $50 \mathrm{~nm}$ silver NPs (a, b and c) with both 2D and 3D projections, imaged using tilt-series TEM tomography. 2D binary projections of viewed down the $z$ axis (left) and 3D images rendered in Tomviz 1.3.0, ${ }^{8}$ as viewed down the $x, y$ and $\mathrm{z}$ axes (right) from left to right. Image taken with permission from ref 9.

\section{Nanoparticle Porosity and Heterogeneity}

If we have a sample of nominally 'spherical' nanoparticles, the question arises as how spherical are they and how much do their shapes and sizes vary across the population? This is certainly an important question where for both large ${ }^{10}$ and small ${ }^{11}$ nanoparticles the thermodynamic shape is not that of a sphere. However, especially when particles have been grown under kinetic control, their shape - although approximately spherical - can in reality vary significantly across a sample. ${ }^{9}$ As an example, Figure 1 presents reconstructions of three nominally spherical silver $(50 \mathrm{~nm})$ particles as viewed down three different axis, showing how for this chemically synthesised material the size and shape of the individual particles varies significantly. Imaging is a key aspect of 
characterising a new material; however, unsurprisingly, the amount of detail we see depends on the resolution of the technique. Figure 2 a) presents a scanning electron microscope image of a commercial $30 \mathrm{~nm}$ platinum nanoparticle sample, here the structures look essentially solid and quasi-spherical. However, transmission electron microscopy reveals this to be far from the truth (Figure $2 \mathrm{~b}$ ). These particles actually comprise of an aggregate of small crystalline structures. Platinum group metals, in contrast to both silver and gold, have a greater propensity to form larger nano-structures via an aggregation growth pathway ${ }^{12}$ leading to complex higher order structures. Furthermore, although it is the 'gold standard' conventional transmission electron microscopy is not without its limitations. First, among other problems, it generally only provides $2 \mathrm{D}$ projections of the $3 \mathrm{D}$ objects under study. Second, the measurement of 'large' sample populations is often prohibitively time expensive. Third, only electron transparent samples can be studied using the technique. In the case of the first problem this is of distinct importance when considering structures akin to those shown in Figures 2. From a heterogeneous catalysis perspective further questions of, how porous these platinum structures are and what the accessible surface area (internal and external) of the material is, are not easily resolved.

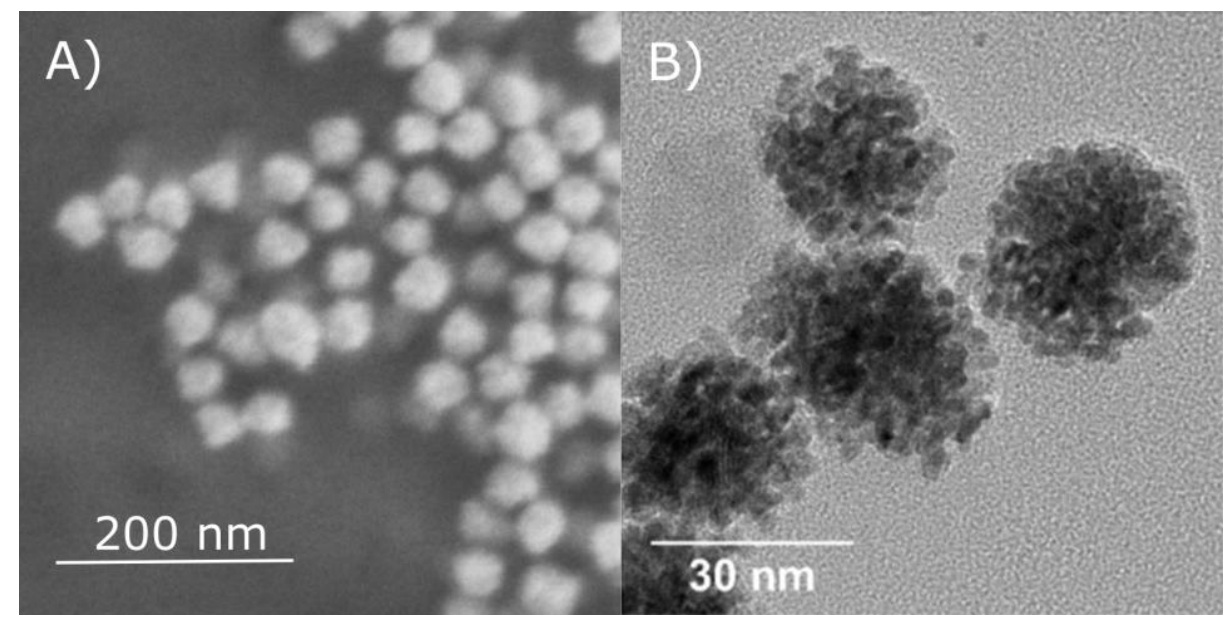

Figure 2: Electron microscope images of $30 \mathrm{~nm}$ platinum nanoparticles (nanoComposix) imaged using a) scanning electron microscopy and b) transmission electron microscopy. Image taken with permission from ref 13. 

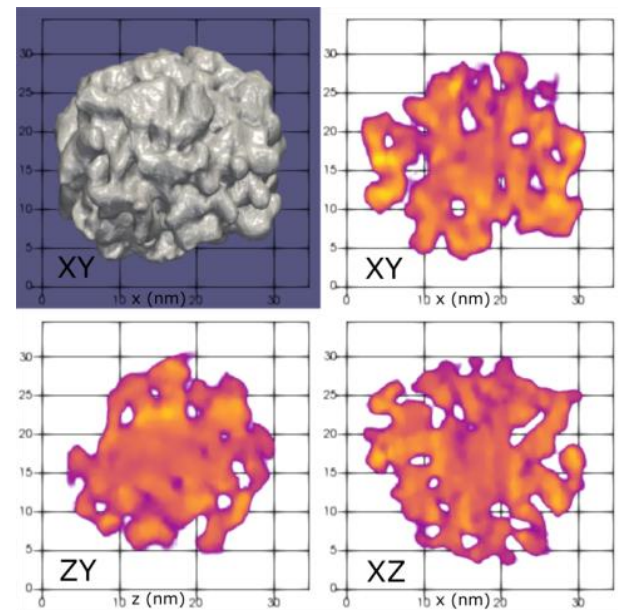

Figure 3: Tomogram reconstruction of the nominally $30 \mathrm{~nm}$ platinum nanoparticles (nanoComposix). Top left: rendered view of the 3D reconstruction viewed down the $\mathrm{z}$-axis. Top right, bottom left and bottom right represent perpendicular cross-sectional views through the nanoparticle in the $X Y, Z Y$ and XZ planes respectively. Image taken with permission from ref 14 .

This characterisation problem is compounded by the fact that conventional surface area measurement techniques are often inapplicable for studying nanomaterials. ${ }^{14}$ Tilt series electron tomography provides one route by which the 3D structure of the particle can be evidenced, this technique relies on taking multiple images of the same particle viewed at a range of angles and the subsequent computer reconstruction of the full $3 \mathrm{D}$ structure. ${ }^{9}$ Figure 3 presents a tomogram reconstruction of these $30 \mathrm{~nm}$ platinum nanoparticles, by looking at slices through the $3 \mathrm{D}$ structure it is revealed that this material is porous throughout ${ }^{14}$ It should be noted that this tendency of larger platinum nanoparticles to be comprised of smaller aggregates is a regular feature of the material and occurs in both commercial ${ }^{15-16}$ and lab ${ }^{17-20}$ synthesised samples. Such materials are variously described as porous, dendritic or star-like and the extent to which these particles, either between batches or synthesis methods, exhibit differing degrees of porosity may plausibly be an important and unaccounted for source of variability in the literature. As an alternative approach to imaging, if the nanoparticles are extremely uniform then the technique of 'single particle reconstruction', as originally developed for organic biological structures, may be applied. ${ }^{21}$ Dick et al. have also recently 
demonstrated an interesting SEM based technique for studying large porous particles (ca. $300 \mathrm{~nm}$ ), where a focused ion beam is used to section the particle and allow both its structure and elemental distribution to be analysed. ${ }^{22}$ However invaluable these electron microscopy techniques are, there is still a need for high-throughput nanoparticle characterisation techniques.

Electrochemistry provides one route by which nanoparticle properties can be assessed, a common method is to drop-cast a layer of material onto an electrode surface and analyse the resulting voltammetric response. A novel application of this technique was found in assessing core-shell nanoparticles and determining what fraction of the nanoparticle population have complete ('perfect') as compared partial ('cracked' or 'broken') shells. ${ }^{23}$ The limitation of this ensemble technique is that although it is able to provide information averaged across the nanoparticle population (but see later in this article) it cannot provide direct evidence regarding particle to particle variability. To this end the method of electrochemical single nanoparticle analysis has developed significantly and by making measurements on an individual particle basis it provides a complementary route by which such questions of sample heterogeneity can be experimentally approached. ${ }^{24}$

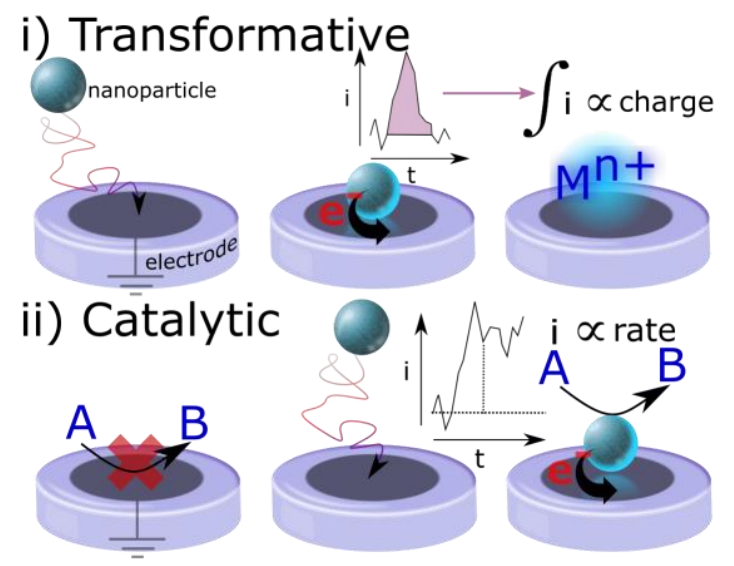

Figure 4: Schematic showing the two main type of nano-impact reactions i) transformative and b) catalytic, where the nanoparticle impacts a microelectrode surface and the resulting current response yields direct information regarding the reaction occurring at the nanoscale. 
Broadly nano-impact responses can be characterised as either i) transformative or ii) catalytic, as schematically outlined in Figure $4 .{ }^{25}$ Other electrochemical nanoparticle detection methods are possible but these are beyond the scope of the present article. ${ }^{25-}$

${ }^{27}$ In the first case (i) when the nanoparticle impacts the potentiostated surface its redox state is changed revealing information regarding the properties of the particle itself. In the second case (ii) the arrival of the nanoparticle serves to catalyse a reaction yielding information regarding the activity of the nanomaterial. The oxidation of individual silver nanoparticles is a prime example of a transformative reaction. ${ }^{28}$ However, some care must be taken in data interpretation to ensure that the nano-reaction has gone to completion. The possibility of an individual nanoparticle impacting an electrode multiple times during a single event was recognised early on ${ }^{29}$ but only experimentally validated ${ }^{30}$ later with the development of sufficiently fast electrochemical measurement devices and with the use of relatively large nanoparticles. For these large multiply impacting particles the nanoparticle oxidation reaction does not necessarily go to completion, leading to an associated loss of information regarding the nanoparticle size distribution. As an alternative example of such a transformative reaction, in 2018 it was demonstrated, in part by the present authors, ${ }^{31-32}$ that the surface areas of individual platinum nanoparticles could be determined by the electrochemical deposition or removal of a monolayer of chemisorbed hydrogen from the catalytic interface. Similar results were also later reported by Zhang et al. ${ }^{33}$. The important result here is that the surface area measurements directly evidence the porous nature of these platinum nanoparticles so giving insight into the surface area size distribution across the population. Furthermore, the extended porous platinum surface of these nanoparticles (shown in Figures 2 and 3) was shown to be chemically accessible and to contribute significantly to the overall activity of the platinum nanomaterial. ${ }^{13}$ A further interesting observation regarding these nano-impact experiments is that in the experimental case of platinum particles impacting a gold surface the nano-material essentially irreversibly adsorbs to the interface for the duration of the experiment; as evidenced by the measured steps 'up' in the catalytic current, with no observation of characteristic steps 'down' as would be associated with the loss of material from the surface. In direct contrast, in the case of gold nanoparticles at a carbon surface the residency time of the nanomaterial is significantly less. ${ }^{34}$ 
Early work ${ }^{17,35}$ in the field on electrocatalytic nano-impact reactions often concluded the electrochemical processes to be limited by the mass-transport of the reagent to the nanoparticle interface (commonly hydrazine to platinum). More recent experiments have served to evidence how the catalytic reaction may, under some circumstances, be limited not by the rate of mass-transport to the catalytic material but by the rate of the interfacial reaction. ${ }^{31}$ This is quite a stark contrast and could simply reflect the differing electrochemical rate constants for the reactions involved; alternatively, the key insight, as first demonstrated by Koper et al. ${ }^{36}$ in relation to the nano-impact experiments, is that higher electrolyte ${ }^{37}$ and hydrazine concentrations - as employed in many experimental setups - leads to extensive agglomeration of the nanoparticles, hence the observed electrochemical responses may not relate to individual nanoparticles but clusters. The use of high electrolyte concentrations is not compulsory ${ }^{31}$ when studying electrochemical reactions at the nanoscale, although there are exceptions; certainly for the oxidation of silver nanoparticles the halide concentration is important. ${ }^{38-39}$ However, as with microelectrodes the mass-transport profile to an individual nanoparticle is convergent and hence under such steady-state mass-transport conditions the electrolyte concentration likely only needs to be double that of the analyte to ensure the reaction is essentially fully supported. ${ }^{40}$ Furthermore, as the dimension of the nanoparticle becomes comparable to that of the Debye length one might expect the influence of migration to become less ${ }^{41}$ not more important.

$$
\text { Nano-impacts have been applied to the study of single enzymes. }{ }^{42-43} \text { Whilst the classical }
$$
Michaelis-Menten kinetics, and related schemes, are well known to describe their catalytic activity, recent fluorescence data ${ }^{44}$ has shown that whilst the MichaelisMenten model describes the time average behaviour of an ensemble of enzymes, individual enzymes may have periods of intense activity separated by periods of relative dormancy. Only on a time average basis is the Michaelis-Menten ensemble response recovered. ${ }^{44}$ Nano-impacts have shown similar behaviour ${ }^{45}$ for the enzyme catalase applied to the decomposition of hydrogen peroxide where the product, oxygen, was detected electrochemically when a single enzyme was close to the electrode surface. This represents an electrochemical demonstration of the time variation of an 'enzymatic' nanoparticle. Further the observation, more generally, prompts the question as to how the electrochemical response of an ensemble reflects that of the 
individual particles. The catalase experiments reveal this in terms of time; in the next section the changed behaviour seen in spatially extensive ensembles is considered.

\section{Porous Electrode Layers}

Electrochemists usually adopt one of two limiting cases to explore nanoparticle electrocatalysis. As related above, in the nano-impact experiment, single particles can be interrogated and if the experiment is repeated many times an average behaviour can be inferred. In the other limit, widely adopted not least due to the extreme ease of implementation, is the formation of drop-cast layers of particles on the surface of an electrode, with voltammetry conducted at the latter and used to infer ensemble average responses. The simplicity of the approach however none the less requires experimental
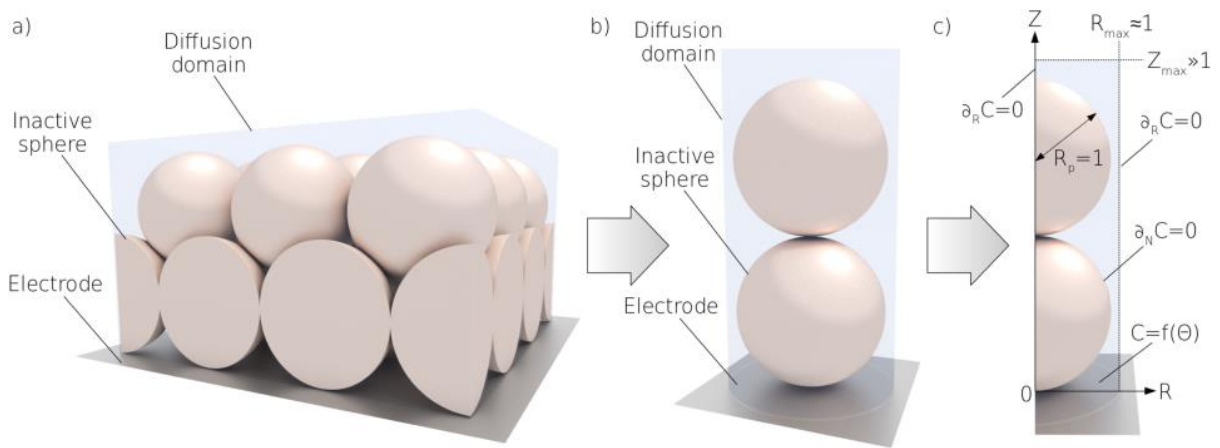

Figure 5: Illustration of the geometry considered. (a) The electrode surface is decorated with inactive nanoparticles arranged in a hexagonal packing pattern. (b) The structure depicted in (a) is approximated as a stack of spheres located centrally in a cylindrical diffusion domain. (c) The rotational symmetry of the diffusion domain is exploited to transform the three-dimensional structure into a two-dimensional geometry over which diffusion equation is solved subject to the boundary conditions shown. $R$ and $Z$ signify dimensionless cylindrical coordinates where the real distances $r$ and $z$ are normalized to the particle radius, $C$ is the dimensionless concentration of the reactant normalized against the bulk concentration, $R_{p}$ is the dimensionless particle radius, $\theta$ is the dimensionless electrode potential, and $f(\theta)$ represents a Nernstian Dirichlet-type boundary condition. The dimensionless radius of the simulated space, $R_{\max }$, is set to 1.05 in all calculations while $Z_{\max }$ is chosen to be a sufficiently large value so as to not perturb the simulation results. Image taken with permission from ref 6. 
care and interpretative due diligence. For example if small, sub monolayer, quantities of nanoparticles are drop-casted, from a suspension followed by evaporation then there is very often a tendency to form aggregates/agglomerates of particles on drying. ${ }^{46}$ Thus the study is that of a low coverage of particle clumps, often ensembles of different sizes thus mirroring a problem mentioned in the previous section in the solution phase. A second issue arises if a multilayer, or a composite, of material is studied. Here, a key issue, in the case of conductive particles, is the extent of electrical contact between the particles within the film. At the very least experiments with different film thicknesses are required. Similarly the extent to which the solvent plus electrolyte ingress into the porous layer may need consideration. The latter consideration prompts the question as to how a porous layer of particles responds voltammetrically. The latter generically reflects in almost all cases a balance between mass-transport and electron transfer kinetics. The former is far from simple as the following hypothetical example shows. In particular the well-known response of a macroelectrode in the form of a flat surface under the influence of linear diffusion has been contrasted, via simulation, ${ }^{6}$ with that of the same electrode modified with a close packed layer of non-conducting spheres (see Figure 5) which are by hypothesis, entirely inert except for altering mass-transport to and from the electrode. Further the interfacial reaction was assumed by definition to be a Nernstian one electron process,

$\mathrm{A}+\mathrm{e} \rightleftharpoons \mathrm{B}$

and pinned as such in the simulation. Thus any observed deviation can only be as a result of the altered mass-transport. Figure 6 shows that the voltammetric peak potential and peak-to-peak separation together with the expected results for a bare electrode. The response is surprisingly complex!

Only at very high scan rates and very low scan rates is the expected response seen. These limits correspond to either diffusion from the solvent immediately adjacent to the electrode surface or to the case where the diffusion layer extends far into the bulk solution such that the porous layer has a negligible effect. At fast scan rates but slower than the very fast, a thin-layer type behaviour is seen corresponding to electrolysis of the layer of the solvent trapped between the electrode and the first layer of spheres. 

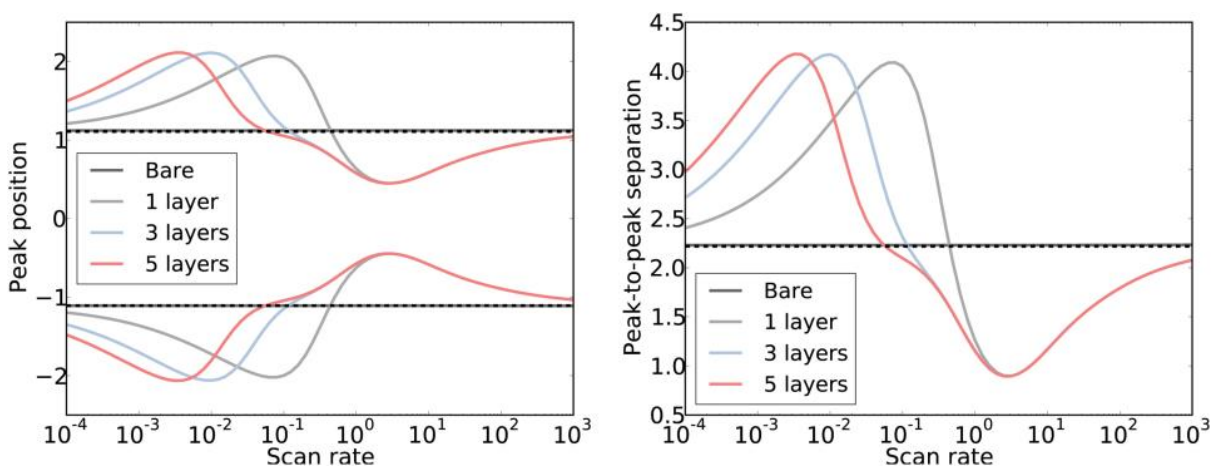

Figure 6: Dimensionless peak positions and peak-to-peak separations as a function of the dimensionless scan rate, where the dimensionless potential is the potential referenced to the formal potential and scaled by F/RT and dimensionless time is $D t / r^{2}$ where $D$ is the diffusion coefficient and $r$ is the particle radius. Dashed black lines indicate the theoretical results for a bare electrode. The voltammetric scan covers a potential range of 20 . Image taken with permission from ref 6 .

Thus the peaks are closer together than expected in the pure one dimensional diffusion limit. However, at slower scan rates, as the diffusion layer extends into the second and other layers of spheres the peaks become further apart than expected for diffusion to a bare electrode. This is because the material 'trapped' in the pockets between the layers suffers retarded diffusion to the electrode surface because of the relatively small size of the 'holes' in the close packed layer through which diffusion must take place. Thus as the scan rate changes from slow to fast the mass-transport changes from linear diffusion to retard diffusion, to retarded diffusion plus thin-layer, to thin-layer, and finally back to linear diffusion.

These observations are important since in the assessment of possible electro-catalytic materials voltammetric peak-to-peak separations are usually used to interpret catalytic effects, positive or negative. In this extreme but very simple example presented, apparent catalysis of both types can be seen simply by altering the mass-transport of the analyte to an electrode surface through the porous layer. At all times the electrode reaction in this simulation is Nernstian by hypothesis so there cannot possibly be any 'electrocatalysis' despite the variation in the peak potentials. Furthermore these effects have been observed experimentally ${ }^{47}$ using for example the oxidation of ferrocene 
methanol. The analysis has been extended to electrochemically irreversible systems ${ }^{48}$ and verified using the oxidation of hydrazine.

Analysis modelling the case of a layer of conducting spherical particles with close packing on an electrode surface shows that, for the case of reversible voltammetry and at the timescales relevant to typical cyclic voltammetry, the solution inside the porous layer can be approximated as at electrochemical equilibrium at all time. ${ }^{49}$ This insight enables the current controlling the electrolysis of analyte inside and outside of the porous layer to be decoupled and appear as superimposed thin-layer and RandlesŠevčík features. The relative contribution of each clearly reflects the thickness of the porous layer, again signaling the essential need for experiments investigating layers of different thickness. Once again the system can give illusions of catalysis if the peak-topeak separation are used solely as an indicator.

In going beyond model systems, in addition to the perhaps unexpectedly subtle masstransport, the roles of adsorption and electrostatics need to be addressed as is treated explicitly in ref. 49 for different systems. Further the complexities of particle modified surfaces are compounded when studying rotating disc electrodes ${ }^{50}$ and rotating ring disc electrodes. The limitations especially for multi-step reactions are well known and recognised as clarified by Johnson, ${ }^{51}$ and Qiao ${ }^{52}$ as well as in ref. 50 and as overviewed by Sokolov ${ }^{53}$ despite occasional valiant attempts to the contrary. ${ }^{54}$ The difficulties of electrochemically characterizing porous layers is not surprising. Similar challenges and contradictions are encountered in simply measuring their surface area. Thus whilst BET adsorption measurements are often used they can only realistically quantitatively apply to adsorption isotherms of Type 2 and Type 4 as authoritatively established by the International Organization for Standardization. ${ }^{55}$ This at the very least implies a need of the measurement of full adsorption isotherms. Recent work has stressed the variability between BET and alternative means of surface area characterization, ${ }^{56}$ such as adsorption of methylene blue or of dopamine. Underpinning both the surface area and the electrochemical work is a need for a bottom up - not an empirical Materials Science - approach synergising theory, simulation and experiment. 
In electrocatalysis empiricism is rife. This is not to be negative of the field, many (most?) major scientific discoveries have come about by chance as opposed to developing from rational design. However, at present the problem is not necessarily the empirical approach but a lack of tools to effectively and reliably characterise and quantify the activities of new materials. In many cases this makes comparing results across different groups and experimental designs difficult if not impossible. The often reported 'standard electrochemical rate constant' may in many cases be viewed as little more than a fitting parameter, the value of which is sensitive to the used analysis model. As evidenced in the simulations above, in the absence of accounting for electrode heterogeneity, roughness and porosity the physical significance of the 'standard electrochemical rate constant' as measured from the position of a voltammetric peak is arguably limited. The complexities and nuances of electrochemistry means that even for relatively simple reactions, determining the operative mechanism and decoupling the kinetics from the influence of mass-transport can be a significant undertaking. The magnitude of this challenge has likely led to some overly bold claims to be made in the literature. Consequently, as a way forward, approaches that allow not just an average response to be reported but enable heterogeneity across a population to be evidenced will likely not only aid our fundamental understanding but will also provide new routes by which we can reliably characterise and study newly synthesised materials. These nanoscale electrochemistry investigations need to be undertaken with the concomitant uptake and development of new electron microscopy techniques for investigating structures at the atomic scale and with computational modelling ranging from (rigorous) DFT calculations to simulation of transport and electrostatics. 


\section{Acknowledgements}

We would like to thank Dr.s E. Kätelhön and L. Chen for insightful discussions and input on the influence of layer porosity on electrochemical responses. Further, the help, input and collaboration of Dr. N. P. Young has been invaluable in exploring the capabilities and limitations of transmission electron microscopy. To this end we would also like to thank Mr. C Little for his extensive intellectual input and work on reconstructing the silver nanoparticle tomograms.

\section{References}

1. Langmuir, I., Transactions of the Faraday Society 1922, 17, 607-620.

2. Ertl, G., Angewandte Chemie International Edition 2008, 47, 3524-3535.

3. Clavilier, J.; Faure, R.; Guinet, G.; Durand, R., Journal of Electroanalytical 355 Chemistry and Interfacial Electrochemistry 1980, 107, 205-209.

4. Climent, V.; Feliu, J. M., Journal of Solid State Electrochemistry 2011, 15, 1297.

5. Eloul, S.; Compton, R. G., Journal of Physical Chemistry C 2015, 119, 2754027549.

6. Kätelhön, E.; Chen, L.; Compton, R. G., Applied Materials Today 2019, 15, 139-

7. Le, H.; Lin, C.; Kätelhön, E.; Compton, R. G., Electrochimica Acta 2019, 298, 778787.

8. M. D. Hanwell, U. A., D. A. Muller and R. Hovden "The Tomviz Project", can be found under https://tomviz.org/,, 2018.

365 9. Little, C. A.; Batchelor-McAuley, C.; Young, N. P.; Compton, R. G., Nanoscale 2018, 10, 15943-15947. ** Reveals through the use of tilt-series transmission electron tomography the shape heterogeneity in a 'spherical' silver nanoparticle sample. Also, intriguingly, indicates the existance of some internal nanoparticle structure, likely formed during the synthesis.

370 10. Sokolov, S. V.; Batchelor-McAuley, C.; Tschulik, K.; Fletcher, S.; Compton, R. G., Chemistry-A European Journal 2015, 21, 10741-10746.

11. Barnard, A. S.; Young, N. P.; Kirkland, A. I.; Van Huis, M. A.; Xu, H., ACS Nano 2009, 3, 1431-1436.

375 P., Science 2009, 324, 1309-1312.

12. Zheng, H.; Smith, R. K.; Jun, Y.-w.; Kisielowski, C.; Dahmen, U.; Alivisatos, A. 13. Yu, W.; Batchelor-McAuley, C.; Chang, X.; Young, N. P.; Compton, R. G., Physical Chemistry Chemical Physics 2019, 21, 20415-20421.

14. Yu, W.; Batchelor-McAuley, C.; Wang, Y.-C.; Shao, S.; Fairclough, S. M.; Haigh, S. J.; Young, N. P.; Compton, R. G., Nanoscale 2019, 11, 17791-17799. * An extensive investigation into the structure of platinum nanoparticles using HAADF STEM tomography to reveal the internal particle structure.

15. Dasari, R.; Robinson, D. A.; Stevenson, K. J., Journal of the American Chemical Society 2013, 135, 570-573. 
16. Xiang, Z.; Deng, H.; Peljo, P.; Fu, Z.; Wang, S.; Mandler, D.; Sun, G.; Liang, Z., Angewandte Chemie 2018, 130, 3522-3526.

17. Xiao, X.; Fan, F.-R. F.; Zhou, J.; Bard, A. J., Journal of the American Chemical Society 2008, 130, 16669-16677.

18. Zhou, H.; Park, J. H.; Fan, F.-R. F.; Bard, A. J., Journal of the American Chemical Society 2012, 134, 13212-13215.

390 19. Tu, Y.; Peng, F.; Sui, X.; Men, Y.; White, P. B.; van Hest, J. C. M.; Wilson, D. A., Nature Chemistry 2017, 9, 480.

20. Bigall, N. C.; Härtling, T.; Klose, M.; Simon, P.; Eng, L. M.; Eychmüller, A., Nano Letters 2008, 8, 4588-4592.

21. Wang, Y.-C.; Slater, T. J. A.; Leteba, G. M.; Roseman, A. M.; Race, C. P.; Young,

395 N. P.; Kirkland, A. I.; Lang, C. I.; Haigh, S. J., Nano Letters 2019, 19, 732-738.

22. Glasscott, M. W.; Pendergast, A. D.; Choudhury, M. H.; Dick, J. E., ACS Applied Nano Materials 2018, 2, 819-830.

23. Tschulik, K.; Ngamchuea, K.; Ziegler, C.; Beier, M. G.; Damm, C.; Eychmueller, A.; Compton, R. G., Advanced Functional Materials 2015, 25, 5149-5158.

400 24. Gooding, J. J., Angewandte Chemie International Edition 2016, 55, 12956-12958. 25. Stevenson, K. J.; Tschulik, K., Current Opinion in Electrochemistry 2017, 6, 3845. * An excellent overview of the nano-impacts field.

26. Sokolov, S. V.; Eloul, S.; Kätelhön, E.; Batchelor-McAuley, C.; Compton, R. G., Physical Chemistry Chemical Physics 2017, 19, 28-43.

405 27. Goines, S.; Dick, J. E., Journal of The Electrochemical Society 2020, 167, 037505. 28. Zhou, Y. G.; Rees, N. V.; Compton, R. G., Angewandte Chemie - International Edition 2011, 50, 4219-4221.

29. Dickinson, E. J. F.; Rees, N. V.; Compton, R. G., Chemical Physics Letters 2012, 528, 44-48.

410 30. Oja, S. M.; Robinson, D. A.; Vitti, N. J.; Edwards, M. A.; Liu, Y.; White, H. S.; Zhang, B., Journal of the American Chemical Society 2017, 139, 708-718.

31. Jiao, X.; Batchelor-McAuley, C.; Lin, C.; Kätelhön, E.; Tanner, E. E. L.; Young, N. P.; Compton, R. G., ACS Catalysis 2018, 8, 6192-6202. ** First reported measurement of the underpotential deposition of hydrogen at individual 415 impacting platinum nanoparticles, thus providing a measure of the particle's surface area. This work also demonstrates that nano-impacts can under some circumstances be studied in low ionic strength media.

32. Jiao, X.; Batchelor-McAuley, C.; Young, N. P.; Compton, R. G., Physical Chemistry Chemical Physics 2018, 20, 23847-23850.

420 33. Defnet, P. A.; Han, C.; Zhang, B., Analytical Chemistry 2019, 91, 4023-4030. * An important and valuable independent corroboration of the Hupd measurements made at individual platinum nanoparticles. Slight care needs to be taken as a numerical error seems to have been made in the conversion of charge to surface area (see Table 1 in the paper by Defnet et al.); furthermore, even once this issue has been corrected for the reported surface areas are larger than would be anticipated on the basis of electron tomography (see ref. 14), plausibly indicating that even with the precautions used agglomeration remains a problem for the technique. Issues raised regarding differences in Hupd surface coverages at differing $\mathrm{pH}$ are likely spurious. 
34. Ly, L. S. Y.; Batchelor-McAuley, C.; Tschulik, K.; Kätelhön, E.; Compton, R. G., Journal of Physical Chemistry C 2014, 118, 17756-17763.

35. Xiao, X.; Bard, A. J., Journal of the American Chemical Society 2007, 129, 9610 9612.

36. Kleijn, S. E. F.; Serrano-Bou, B.; Yanson, A. I.; Koper, M. T. M., Langmuir 2013, 29, 2054-2064.

37. El Badawy, A. M.; Scheckel, K. G.; Suidan, M.; Tolaymat, T., Science of the Total Environment 2012, 429, 325-331.

38. Krause, K. J.; Brings, F.; Schnitker, J.; Kätelhön, E.; Rinklin, P.; Mayer, D.; Compton, R. G.; Lemay, S. G.; Offenhäusser, A.; Wolfrum, B., Chemistry-A European Journal 2017, 23, 4638-4643.

39. Ngamchuea, K.; Clark, R. O. D.; Sokolov, S. V.; Young, N. P.; BatchelorMcAuley, C.; Compton, R. G., Chemistry-A European Journal 2017, 23, 16085-16096.

40. Batchelor-McAuley, C.; Ngamchuea, K.; Compton, R. G., Journal of Electroanalytical Chemistry 2018, 830, 88-94.

445 41. Guo, Y.; Yang, M.; Batchelor-McAuley, C.; Compton, R. G., Journal of Electroanalytical Chemistry 2019, 849, 113370.

42. Sekretaryova, A. N.; Vagin, M. Y.; Turner, A. P. F.; Eriksson, M., Journal of the American Chemical Society 2016, 138, 2504-2507. * Important initial report of enzyme impacts but lacks analysis of the frequency and size of the events.

43. Kätelhön, E.; Sepunaru, L.; Karyakin, A. A.; Compton, R. G., ACS Catalysis 2016, $6,8313-8320$

44. Flomenbom, O.; Velonia, K.; Loos, D.; Masuo, S.; Cotlet, M.; Engelborghs, Y.; Hofkens, J.; Rowan, A. E.; Nolte, R. J. M.; Van der Auweraer, M., Proceedings of the National Academy of Sciences 2005, 102, 2368-2372.

45. Lin, C.; Sepunaru, L.; Kätelhön, E.; Compton, R. G., Journal of Physical Chemistry Letters 2018, 9, 2814-2817. * Enzymic impacts where both the frequency and size of the events has been fully addressed.

46. Toh, H. S.; Batchelor-McAuley, C.; Tschulik, K.; Uhlemann, M.; Crossley, A.; Compton, R. G., Nanoscale 2013, 5, 4884-4893.

47. Chen, L.; Kätelhön, E.; Compton, R. G., Applied Materials Today 2019, 16, 141145.

48. Chen, L.; Kätelhön, E.; Compton, R. G., Applied Materials Today 2019, 100480.

49. Kätelhön, E.; Compton, R. G., Applied Materials Today 2020, 18, 100514.

50. Masa, J.; Batchelor-McAuley, C.; Schuhmann, W.; Compton, R. G., Nano

465 Research 2014, 7, 71-78.

51. Treimer, S.; Tang, A.; Johnson, D. C., Electroanalysis 2002, 14, 165-171.

52. Zhou, R.; Zheng, Y.; Jaroniec, M.; Qiao, S.-Z., ACS Catalysis 2016, 6, 4720-4728.

* Most persuasively cautions against the use of the Koutecky-Levich method for analysing rotating disc electrode data.

470 53. Sokolov, S. V.; Sepunaru, L.; Compton, R. G., Applied Materials Today 2017, 7, $82-90$.

54. Guo, S.-X.; Zhao, S.-F.; Bond, A. M.; Zhang, J., Langmuir 2012, 28, 5275-5285.

55. International Organization for Standardization. Technical Committee Iso/Tc 24. Particle characterization including sieving, Determination of the Specific Surface Area of Solids by Gas Adsorption: BET Method. ISO 9277:2010: 2010. 
56. Chen, L.; Batchelor-McAuley, C.; Rasche, B.; Johnston, C.; Hindle, N.; Compton, R. G., Applied Materials Today 2020, 18, 100506. * Critically compares the use of BET and other methods for the measurement of the surface areas of carbon materials. Introduces dopamine as an alternative to the classical methylene blue surface area method. 